A. Pramesh Rao, G. Swarup and Gopal-Krishna, eds.

\title{
Giant Radio Galaxies and the Inter Galactic Medium
}

\author{
Arno P. Schoenmakers \\ N.F.R.A., P.O. Box 2, 7990-AA, Dwingeloo, The Netherlands, \\ Astronomical Institute Utrecht, P.O. Box 80000, 3508-TA Utrecht, \\ and Sterrewacht Leiden, P.O. Box 9500, 2300-RA Leiden, \\ The Netherlands
}

A.G. de Bruyn

N.F.R.A., P.O. Box 2, 7990-AA, Dwingeloo, The Netherlands, and Kapteyn Astronomical Institute, P.O. Box 900, 9700-AV, Groningen, The Netherlands

\section{H.J.A. Röttgering}

Sterrewacht Leiden, P.O. Box 9500, 2300-RA Leiden, The Netherlands

H. van der Laan

Astronomical Institute Utrecht, P.O. Box 80000, 3508-TA Utrecht, The Netherlands

\begin{abstract}
Using the WENSS survey, we have selected a complete sample of 26 Giant Radio Galaxies. We have investigated the properties of their radio structures using multifrequency radio observations. A spectral ageing analisys reveals that, among other things, that these sources are typically $50-100 \mathrm{Myr}$ old and that their lobes have higher advance velocities than smaller sources of similar radio power. Further, we find pressure gradients in their radio lobes, suggesting that the lobes are still overpressured with respect to the environment. We find no evidence for a cosmological evolution of the radio lobe pressures with increasing redshift, at least up to $z \sim 0.3$, other than that caused by our selection technique. A faint, higher redshift sample of giant sources is needed to constrain the pressure in their environments, the IGM. We are well underway with selecting such a sample, and the GMRT can play an important role in studying the radio properties of these sources.
\end{abstract}

\section{Introduction}

Giant radio galaxies (GRGs) are radio sources whose lobes span a (projected) distance of above $1 \mathrm{Mpc}\left(H_{0}=50 \mathrm{~km} \mathrm{~s}^{-1} \mathrm{Mpc}^{-1}, q_{0}=0.5\right)$. Since radio sources grow in size as they get older (e.g. Scheuer 1974, Baldwin 1982), GRGs must represent a late phase in the evolution of radio sources. 
Not all radio sources will live long enough, or grow rapidly enough, to reach the size of the Giant radio sources. What fraction of these do and under what circumstances is still largely unclear. Radio source evolution models predict that GRGs are extremely old (i.e. typically above $10^{8} \mathrm{yr}$ ) and/or located in very underdense environments, as compared to smaller radio sources (e.g. Kaiser \& Alexander 1999).

The most commonly used method to estimate the age of a radio source is by means of a spectral ageing analysis (e.g. Alexander \& Leahy 1987). The first systematically obtained results of a small, incomplete sample of GRGs show that these spectral ages are indeed comparable to those predicted by the models (Mack et al. 1998).

By studying the physical properties of the radio lobes of GRGs we can constrain the properties of the intergalactic medium (IGM). For instance, in an adiabatically expanding Universe filled with a hot, diffuse and uniform IGM, the IGM pressure, $p_{i g m}$, should increase as a function of redshift, $z$, as $p_{\text {igm }} \propto$ $(1+z)^{5}$ (e.g. Subrahmanyan \& Saripalli 1993). Using a small sample of GRGs, Subrahmanyan \& Saripalli limit the local value of the IGM pressure, $p_{i g m, 0}$, to $p_{i g m, 0} \lesssim 2 \times 10^{-14}$ dyn $\mathrm{cm}^{-2}$ by equalling it to the radio lobe equipartition pressure. Cotter (1998) shows that the observed evolution in GRG radio lobe pressures does not contradict a $(1+z)^{5}$ evolution, at least out to $z \sim 1$. However, these results might be biased since the known higher redshift GRGs are the most powerful ones at their epochs and thus are expected to have the highest equipartition lobe pressures.

In order to address the above mentioned issues more carefully, it is vital to use a sample of GRGs with well understood selection effects. We have compiled such a sample from the 325-MHz Westerbork Northern Sky Survey (WENSS). We have selected all radio sources with a (projected) size exceeding $1 \mathrm{Mpc}$, a flux density above $1 \mathrm{Jy}$, an angular size above 5 arcminutes and a distance from the galactic plane larger than 12.5 degrees. This complete sample consists of 26 sources, of which 10 are newly discovered GRGs (Schoenmakers 1999).

\section{Spectral ages and radio lobe pressure evolution}

Using WENSS, the 1.4-GHz NRAO VLA Sky Survey (NVSS) and new 10.5-GHz Effelsberg observations we have determined the properties of the radio lobes of the 22 FRII-type (Fanaroff \& Riley 1974) sources in our sample. In cases where we could determine the spectral age we find ages which are in the range of 50 $100 \mathrm{Myr}$, which agrees with earlier results and model predictions. We also find that the GRGs tend to have higher lobe advance velocities than smaller sources of similar observed radio power (by a factor of two, typically; Schoenmakers 1999).

For the FRII-type sources which are well resolved by our observations (i.e. larger than 8 arcminutes) we have measured the radio lobe pressure along the radio axes. The majority of our sources show a decrease in energy density when going back from the hotspots to the radio cores. In some cases, the energy density rises again in the vicinity of the center, probably due to the presence of a strong radio core and/or jet, or to the presence of a denser galactic halo or cluster core. Since the pressure in the IGM at distances of a few hundred kpc 


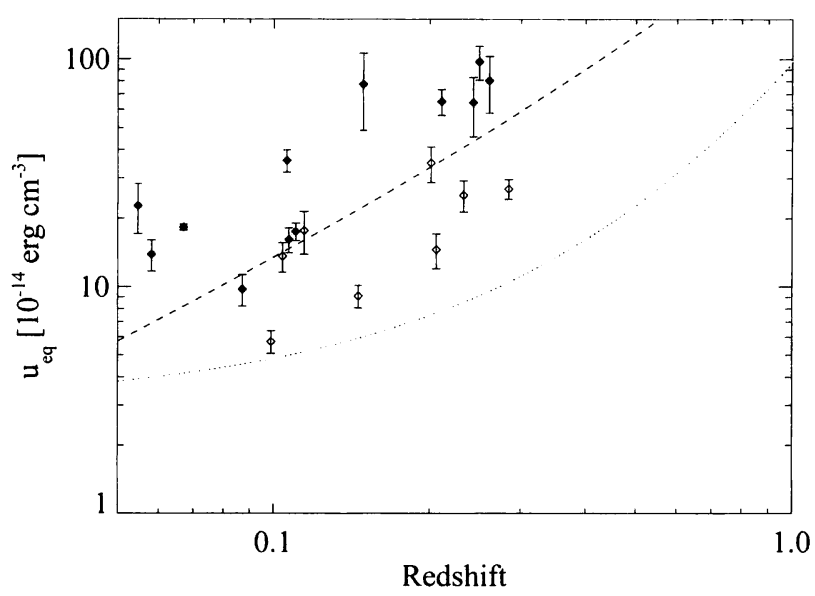

Figure 1. The intensity weighted average equipartition energy density in the radio lobes of the FRII-type GRGs in our sample against redshift. Filled symbols: Linear size between 1 and 2 Mpc. Open symbols: Linear size $>2$ Mpc. Dashed line: The expected energy density of a source with a flux density, volume and spectral index as given by the median values of these properties in our sample, i.e. the relation expected on basis of our selection criteria. Dotted line: Expected relation if the lobes are in pressure equilibrium with the IGM and $p_{i g m}(z)=1.0 \times 10^{-14} \cdot(1+z)^{5}$ dyn $\mathrm{cm}^{-2}$.

from the radio core should be roughly constant, the observed pressure gradients in the lobes suggest that the lobes are overpressured with respect to the ambient medium.

Figure 1 shows the intensity weighted average lobe energy density against the redshifts of the sources. We have made a separation between sources which are smaller and larger than $2 \mathrm{Mpc}$ (closed and open symbols, respectively). There are two things to note in this plot. First, the larger sources tend to have the lowest average lobe pressures. This agrees with model predictions for radio source evolution (e.g. Kaiser \& Alexander 1997). Second, we find a correlation between lobe energy density and redshift, in agreement with Saripalli \& Subrahmanyan (1993) and Cotter (1998). Note that the found correlation does not contradict a $(1+z)^{5}$ increase of the IGM pressure, provided that the current day value is $\lesssim 10^{-14}$ dyn $\mathrm{cm}^{-2}$ (indicated by the dotted line in Fig. 1). However, the observed increase in lobe energy density with increasing redshift also matches the expected behaviour for a source of fixed dimensions and flux density, which is indicated by the dashed line in Fig. 1. Therefore, we conclude that the observed increase with redshift is more likely due to the use of a flux density and source volume limited sample than to any cosmological effect. This applies to the giant sources in Cotter (1998) as well, which are mostly from a flux density limited sample (flux density between 0.4 and $1.0 \mathrm{Jy}$ at $151 \mathrm{MHz}$ ) of sources larger than $\sim 1 \mathrm{Mpc}$. There is therefore no evidence for a strong increase in the IGM pressure with increasing redshift in the current data. 


\section{High redshift GRGs}

To investigate whether the IGM pressure truly evolves as strongly as $(1+z)^{5}$, it will be necessary to find Mpc-sized radio sources at higher redshifts $(z>0.4)$. The existence of a population of GRGs with lobe energy densities of $\lesssim 3 \times 10^{-14}$ erg $\mathrm{cm}^{-3}$ at redshifts of at least 0.6 would be difficult to reconcile with a strong IGM pressure evolution, unless the current day IGM pressure is much lower than $10^{-14}$ dyn $\mathrm{cm}^{-2}$. Such sources are expected to have flux densities of $\lesssim 200$ $\mathrm{mJy}$ at $325 \mathrm{MHz}$ (assuming a size of $1.5 \mathrm{Mpc}$, a spectral index of -0.8 and an aspect ratio of 3), and should therefore be present in WENSS. We have started to compile a sample of higher redshift GRGs, which at this point contains 30 GRGs with $z>0.4$ (see Schoenmakers et al. 1998 for details and preliminary results). The highest redshift GRG we have found yet has a (tentative) redshift of 2.74 .

High redshift GRGs are interesting in many other respects as well. For instance, they can be used to investigate the effect of the highly increased Inverse Compton losses on the radio spectrum of the lobes, to study radio source evolution as a function of cosmic epoch and to trace low density regions of the high redshift IGM. Detailed observations of their radio structures at low frequencies (i.e. $<1 \mathrm{GHz}$ ) are vital to correctly estimate the lobe energy densities and map the spectral indices and rotation measures over the extended radio lobes. The GMRT provides low frequency capabilities at angular resolutions which are sufficient for the study of high redshift GRGs (angular sizes $\gtrsim 1$ arcmin) and will therefore become an important instrument in our future studies of these sources.

Acknowledgments: A.P.S. kindly acknowledges the I.A.U. for financial support and NCRA/IUCAA for the offered hospitality during his stay in India.

\section{References}

Alexander, P., \& Leahy, J. P. 1987, MNRAS, 225, 1

Baldwin, J. E. 1982, in "Extragalactic radio sources", Ed. D. S. Heeschen \& C. M. Wade (Dordrecht: D. Reidel Publishing Co.), 21

Cotter, G. 1998, "Observational cosmology with the new radio surveys", Ed. M.

N. Bremer, N. Jackson \& I. Pérez-Fournon (Dordrecht: Kluwer), 233

Fanaroff, B. L., \& Riley, J. M. 1974, MNRAS, 167, 31

Kaiser, C. R., \& Alexander, P. 1997, MNRAS, 286, 215

Kaiser, C. R., \& Alexander P. 1999, MNRAS, 305, 707

Mack, K.-H., Klein, U., O'Dea, C. P., Willis, A. G., Saripalli L. 1998, A\&A, 329,431

Scheuer, P. A. G. 1974, MNRAS, 166, 513

Schoenmakers, A. P., Röttgering, H. J. A, van der Laan, H., de Bruyn, A.G. 1998, "Observational cosmology with the new radio surveys", Ed. M. N.

Bremer, N. Jackson \& I. Pérez-Fournon (Dordrecht: Kluwer), 239

Schoenmakers, A. P. 1999, Ph.D.-Thesis, Utrecht University

Subrahmanyan, R., \& Saripalli, L. 1993, MNRAS, 260, 908 